PDES, SUBMANIFOLDS AND

AFFINE DIFFERENTIAL GEOMETRY

BANACH CENTER PUBLICATIONS, VOLUME 69

INSTITUTE OF MATHEMATICS

POLISH ACADEMY OF SCIENCES

WARSZAWA 2005

\title{
ON SOME CLASS OF HYPERSURFACES WITH THREE DISTINCT PRINCIPAL CURVATURES
}

\author{
KATARZYNA SAWICZ \\ Institute of Econometrics and Computer Science \\ Technical University of Częstochowa \\ Armii Krajowej 19B, 42-200 Częstochowa, Poland \\ E-mail: ksawicz@zim.pcz.czest.pl
}

\begin{abstract}
We investigate hypersurfaces $M$ in spaces of constant curvature with some special minimal polynomial of the second fundamental tensor $H$ of third degree. We present a curvature characterization of pseudosymmetry type for such hypersurfaces. We also prove that if such a hypersurface is a manifold with pseudosymmetric Weyl tensor then it must be pseudosymmetric.
\end{abstract}

1. Introduction. Let $M, n=\operatorname{dim} M \geq 3$, be a connected hypersurface in a semiRiemannian manifold $\left(N, g^{N}\right)$. We denote by $g$ the metric tensor induced on $M$ from $g^{N}$. Further, let $H$, resp. $\mathcal{A}$, be the second fundamental tensor, resp. the shape operator, of $(M, g)$ in $\left(N, g^{N}\right)$. It is well known that $H(X, Y)=g(\mathcal{A} X, Y)$, for any vector fields $X$ and $Y$ tangent to $M$. We define the $(0,2)$-tensor $H^{k}, k \geq 1$, by $H^{k}(X, Y)=g\left(\mathcal{A}^{k} X, Y\right)$, where $H^{1}=H$ and $\mathcal{A}^{1}=\mathcal{A}$. In Sections 3 and 4 we present further basic facts relating to hypersurfaces.

A hypersurface $M, n \geq 3$, in $\left(N, g^{N}\right)$ is said to be quasi-umbilical at $x \in M$ if at this point we have

$$
H=\alpha g+\beta u \otimes u, \quad u \in T_{x}^{*} M, \quad \alpha, \beta \in \mathbb{R} .
$$

If $\alpha=0$ (resp., $\beta=0$ or $\alpha=\beta=0$ ) at $x$ then $M$ is called cylindrical (resp., umbilical or geodesic) at $x$. If (1) is fulfilled at every point of $M$ then it is called a quasi-umbilical hypersurface. A hypersurface $M, n \geq 4$, in $\left(N, g^{N}\right)$ is said to be 2-quasi-umbilical at $x \in M$ (see [16] and references therein) if at this point we have

$$
H=\alpha g+\beta u \otimes u+\gamma v \otimes v, \quad u, v \in T_{x}^{*} M, \quad \alpha, \beta, \gamma \in \mathbb{R},
$$

2000 Mathematics Subject Classification: Primary 53B20, 53B25; Secondary 53C25.

Key words and phrases: pseudosymmetric manifold, manifold with pseudosymmetric Weyl tensor, hypersurface, 2-quasi-umbilical hypersurface.

The author is supported by VolkswagenStiftung (Germany).

The paper is in final form and no version of it will be published elsewhere. 
where $U, V$ are vectors at $x$ such that $g(U, V)=0, u(X)=g(U, X)$ and $v(X)=g(V, X)$ for every vector $X$ at $x$. If (2) is fulfilled at every point of $M$ then it is called a 2quasi-umbilical hypersurface. If $\alpha=0$ at $x$ then $M$ is called 2-cylindrical at $x$. It is clear that if $\left(N, g^{N}\right)$ is a Riemannian manifold then the above definition of a 2-quasi-umbilical hypersurface $M$ at $x \in M$ is equivalent to the following: the hypersurface $M, n \geq 4$, in a Riemannian manifold $\left(N, g^{N}\right)$ is said to be 2-quasi-umbilical at $x \in M$ when at $x$ it has a principal curvature with multiplicity $\geq n-2$, i.e. when the principal curvatures at $x$ are $\mu, \nu, \lambda, \ldots, \lambda$, where $\lambda$ occurs $(n-2)$-times. Evidently, 2-quasi-umbilical hypersurfaces form a natural extension of the class of quasi-umbilical hypersurfaces.

Every 2-quasi-umbilical hypersurface in conformally flat space is a manifold with pseudosymmetric Weyl tensor (see Theorem 3.1(ii)). A semi-Riemannian manifold $(M, g)$, $n \geq 4$, is said to be a manifold with pseudosymmetric Weyl tensor ([5], Section 12.6) if, at every point of $M$, the tensors $C \cdot C$ and $Q(g, C)$ are linearly dependent. This is equivalent on $\mathcal{U}_{C}=\{x \in M \mid C \neq 0$ at $x\}$ to

$$
C \cdot C=L_{C} Q(g, C),
$$

where $L_{C}$ is some function on $\mathcal{U}_{C}$. For precise definitions of the symbols used we refer to Sections 2 and 3 of this paper and [3] and [10].

Now let $M$ be a hypersurface in a semi-Riemannian space $N_{s}^{n+1}(c)$ of constant curvature with signature $(s, n+1-s), n \geq 4$, where $c=\frac{\widetilde{\kappa}}{n(n+1)}$ and $\widetilde{\kappa}$ denotes the scalar curvature of the ambient space. We denote by $\mathcal{U}_{H}$ the set of all points of $M$ at which the tensor $H^{2}$ of $M$ is not a linear combination of the metric tensor $g$ and $H$. It is known that $\mathcal{U}_{H} \subset \mathcal{U}_{C} \cap \mathcal{U}_{S}$, where $\mathcal{U}_{S} \subset M$ is defined by $\mathcal{U}_{S}=\left\{x \in M \mid S-\frac{\kappa}{n} g \neq 0\right.$ at $\left.x\right\}$ (see e.g. [10], Section 2). Theorem 4.3 of [17] and Lemma 4.1 and Theorem 4.1 of [4] imply

THEOREM 1.1. If $M$ is a hypersurface with pseudosymmetric Weyl tensor in $N_{s}^{n+1}(c)$, $n \geq 4$, satisfying on $\mathcal{U}_{H} \subset M$ the equation

$$
H^{3}=\operatorname{tr}(H) H^{2}+\psi H,
$$

where $\psi$ is some function on $\mathcal{U}_{H}$, then on this set we have

$$
R \cdot R=\frac{\widetilde{\kappa}}{n(n+1)} Q(g, R) .
$$

Theorem 5.2 of this paper shows that the above theorem remains true if on $\mathcal{U}_{H}$ we replace (4) by

$$
H^{3}=\operatorname{tr}(H) H^{2}+\psi H+\rho g,
$$

where $\psi$ and $\rho$ are some functions on $\mathcal{U}_{H}$. Thus in particular, from Theorem 5.2 it follows that if $M$ is a hypersurface with pseudosymmetric Weyl tensor in a Riemannian space of constant curvature satisfying (6) on $\mathcal{U}_{H} \subset M$ then (5) holds on $\mathcal{U}_{H}$. Further, Theorem 5.3 shows that every hypersurface $M$ in $N_{s}^{n+1}(c), n \geq 4$, satisfying (6) on $\mathcal{U}_{H} \subset M$ is 2-cylindrical on this set.

We remark that if $(6)$ holds on the subset $\mathcal{U}_{H}$ of a hypersurface $M$ in a Riemannian space of constant curvature then at every point of this set $M$ has three three distinct principal curvatures. 
A semi-Riemannian manifold $(M, g), n \geq 4$, is said to be pseudosymmetric ([5], Section 3.1) if, at every point of $M$, the tensors $R \cdot R$ and $Q(g, R)$ are linearly dependent. This is equivalent on $\mathcal{U}_{R}=\left\{x \in M \mid R-\frac{\kappa}{(n-1) n} G \neq 0\right.$ at $\left.x\right\}$ to

$$
R \cdot R=L_{R} Q(g, R),
$$

where $L_{R}$ is some function on $\mathcal{U}_{R}$.

Hypersurfaces satisfying (3), resp., (7), were investigated e.g. in: [15], [16] and [17], resp., in: [2], [4], [6], [7], [11], and [18]. We say that (3) and (7) are certain conditions of pseudosymmetry type. For a recent survey of results on manifolds satisfying such conditions we refer to [3].

Hypersurfaces in semi-Euclidean spaces in $\mathbb{E}_{s}^{n+1}, n \geq 4$, satisfying (6) were investigated in [1]. Examples of such hypersurfaces in $\mathbb{E}_{s}^{n+1}$, with $\rho \neq 0$, will be presented in [19] (see also [13]).

We note that on the set $M \backslash U_{H}$ of a hypersurface $M$ in $N_{s}^{n+1}(c), n \geq 4,(3)$ and (7) are always satisfied (see e.g. [17], Theorem 3.1). Thus in particular, (3) and (7) are satisfied at all points at which $M$ has only two distinct principal curvatures. We present now some results of [2], [4] and [7].

THEOREM 1.2. Let $M$ be a hypersurface in $N_{s}^{n+1}(c), n \geq 4$, and let $\mathcal{U}_{H} \subset M$.

(i) ([2], Theorem 3.1) The conditions (7) and $R \cdot C=L_{R} Q(g, C)$ are equivalent on $\mathcal{U}_{C} \subset M$.

(ii) ([4], Lemma 4.1 and Theorem 4.1) If $M$ is pseudosymmetric then (5) holds on $\mathcal{U}_{H}$. (iii) ([7], Theorems 3.1 and 5.1) The equation rankH $=2$ is satisfied on $\mathcal{U}_{H}$ if and only if (5) holds on this set.

(iv) ([4], Proposition 3.2, Proposition 3.1(ii)) (4) holds on $\mathcal{U}_{H}$ if and only if on this set we have

$$
R \cdot S=\frac{\widetilde{\kappa}}{n(n+1)} Q(g, S)
$$

In connection with the results presented above, we consider in Section 4 the question of finding a condition of pseudosymmetry type equivalent to (6) on the set $\mathcal{U}_{H}$ of a hypersurface $M$ in $N_{s}^{n+1}(c), n \geq 4$. Propositions 5.1 and 5.2 solve this question. Namely we have: (6) holds on $\mathcal{U}_{H} \subset M$ if and only if on this set we have

$$
C \cdot R=L_{1} Q(S, R)+L_{2} Q(g, R)+L_{3} Q(S, G)
$$

for some functions $L_{1}, L_{2}$ and $L_{3}$. Further, let $M$ be a hypersurface in a Riemannian space of constant curvature $N^{n+1}(c), n \geq 4$, and let $V \subset \mathcal{U}_{H} \subset M$ be the set of all points at which the tensor $R \cdot S-\frac{\widetilde{\kappa}}{n(n+1)} Q(g, S)$ is nonzero. Our main result is the following (see Theorem 5.1)

THEOREM 1.3. Let $M$ be a hypersurface in $N_{s}^{n+1}(c), n \geq 4$, and let $V$ be the set defined above. Then on $V$ the condition (6) is equivalent to (33) and (36).

We note that from Theorem 1.2(iv) it follows that if $M$ is a hypersurface of a Riemannian space of constant curvature and (6) holds on $V$, then at every point of this set there are exactly three distinct nonzero principal curvatures. Now Theorem 1.3 implies 
COROLlaRY 1.1. Let $M$ be a hypersurface in a Riemannian space of constant curvature $N^{n+1}(c), n \geq 4$, and let $V$ be the set defined above. Then on $V$ the condition (6) is equivalent to (33) and (36).

The author would like to express her thanks to the referee for his hints, remarks and comments.

2. Preliminaries. Throughout this paper all manifolds are assumed to be connected paracompact manifolds of class $C^{\infty}$. Let $(M, g)$ be an $n$-dimensional, $n \geq 3$, semiRiemannian manifold and let $\nabla$ be its Levi-Civita connection and $\Xi(M)$ the Lie algebra of vector fields on $M$. We define on $M$ the endomorphisms $X \wedge_{A} Y$ and $\mathcal{R}(X, Y)$ of $\Xi(M)$ by

$$
\begin{aligned}
\left(X \wedge_{A} Y\right) Z & =A(Y, Z) X-A(X, Z) Y, \\
\mathcal{R}(X, Y) Z & =\nabla_{X} \nabla_{Y} Z-\nabla_{Y} \nabla_{X} Z-\nabla_{[X, Y]} Z,
\end{aligned}
$$

respectively, where $A$ is a symmetric $(0,2)$-tensor on $M$ and $X, Y, Z \in \Xi(M)$. The Ricci tensor $S$, the Ricci operator $\mathcal{S}$ and the scalar curvature $\kappa$ of $(M, g)$ are defined by

$$
S(X, Y)=\operatorname{tr}\{Z \rightarrow \mathcal{R}(Z, X) Y\}, \quad g(\mathrm{~S} X, Y)=S(X, Y), \quad \kappa=\operatorname{tr} \mathrm{S},
$$

respectively. The endomorphism $\mathcal{C}(X, Y)$ is defined by

$$
\mathcal{C}(X, Y) Z=\mathcal{R}(X, Y) Z-\frac{1}{n-2}\left(X \wedge_{g} \mathrm{~S} Y+\mathrm{S} X \wedge_{g} Y-\frac{\kappa}{n-1} X \wedge_{g} Y\right) Z
$$

Now the $(0,4)$-tensor $G$, the Riemann-Christoffel curvature tensor $R$ and the Weyl conformal curvature tensor $C$ of $(M, g)$ are defined by

$$
\begin{aligned}
& G\left(X_{1}, X_{2}, X_{3}, X_{4}\right)=g\left(\left(X_{1} \wedge_{g} X_{2}\right) X_{3}, X_{4}\right), \\
& R\left(X_{1}, X_{2}, X_{3}, X_{4}\right)=g\left(\mathcal{R}\left(X_{1}, X_{2}\right) X_{3}, X_{4}\right), \\
& C\left(X_{1}, X_{2}, X_{3}, X_{4}\right)=g\left(\mathcal{C}\left(X_{1}, X_{2}\right) X_{3}, X_{4}\right),
\end{aligned}
$$

respectively, where $X_{1}, X_{2}, \ldots \in \Xi(M)$. Let $\mathcal{B}(X, Y)$ be a skew-symmetric endomorphism of $\Xi(M)$ and let $B$ be a $(0,4)$-tensor associated with $\mathcal{B}(X, Y)$ by

$$
B\left(X_{1}, X_{2}, X_{3}, X_{4}\right)=g\left(\mathcal{B}\left(X_{1}, X_{2}\right) X_{3}, X_{4}\right) .
$$

The tensor $B$ is said to be a generalized curvature tensor if

$$
\begin{aligned}
& B\left(X_{1}, X_{2}, X_{3}, X_{4}\right)+B\left(X_{2}, X_{3}, X_{1}, X_{4}\right)+B\left(X_{3}, X_{1}, X_{2}, X_{4}\right)=0, \\
& B\left(X_{1}, X_{2}, X_{3}, X_{4}\right)=B\left(X_{3}, X_{4}, X_{1}, X_{2}\right) .
\end{aligned}
$$

Let $\mathcal{B}(X, Y)$ be a skew-symmetric endomorphism of $\Xi(M)$ and let $B$ be the tensor defined by (10). We extend the endomorphism $\mathcal{B}(X, Y)$ to derivation $\mathcal{B}(X, Y)$. of the algebra of tensor fields on $M$, assuming that it commutes with contractions and $\mathcal{B}(X, Y) \cdot f=0$ for any smooth function $f$ on $M$. Now for a $(0, k)$-tensor field $T, k \geq 1$, we define the $(0, k+2)$-tensor $B \cdot T$ by

$$
\begin{aligned}
(B \cdot T)\left(X_{1}, \ldots, X_{k} ; X, Y\right)=(\mathcal{B}(X, Y) \cdot T)\left(X_{1}, \ldots, X_{k} ; X, Y\right) & \\
& =-T\left(\mathcal{B}(X, Y) X_{1}, X_{2}, \ldots, X_{k}\right)-\cdots-T\left(X_{1}, \ldots, X_{k-1}, \mathcal{B}(X, Y) X_{k}\right) .
\end{aligned}
$$


If $A$ is a symmetric $(0,2)$-tensor then we define the $(0, k+2)$-tensor $Q(A, T)$ by

$$
\begin{aligned}
& Q(A, T)\left(X_{1}, \ldots, X_{k} ; X, Y\right)=\left(X \wedge_{A} Y \cdot T\right)\left(X_{1}, \ldots, X_{k} ; X, Y\right) \\
& \quad=-T\left(\left(X \wedge_{A} Y\right) X_{1}, X_{2}, \ldots, X_{k}\right)-\cdots-T\left(X_{1}, \ldots, X_{k-1},\left(X \wedge_{A} Y\right) X_{k}\right) .
\end{aligned}
$$

In this manner we obtain the $(0,6)$-tensors $B \cdot B$ and $Q(A, B)$. Setting in the above formulas $\mathcal{B}=\mathcal{R}$ or $\mathcal{B}=\mathcal{C}, T=R$ or $T=C$ or $T=S, A=g$ or $A=S$, we get the tensors $R \cdot R, R \cdot C, C \cdot R, R \cdot S, Q(g, R), Q(S, R), Q(g, C)$ and $Q(g, S)$. For symmetric $(0,2)$-tensors $E$ and $F$, we denote their Kulkarni-Nomizu product by

$$
\begin{aligned}
(E \wedge F)\left(X_{1}, X_{2}, X_{3}, X_{4}\right)= & E\left(X_{1}, X_{4}\right) F\left(X_{2}, X_{3}\right)+E\left(X_{2}, X_{3}\right) F\left(X_{1}, X_{4}\right) \\
& -E\left(X_{1}, X_{3}\right) F\left(X_{2}, X_{4}\right)-E\left(X_{2}, X_{4}\right) F\left(X_{1}, X_{3}\right) .
\end{aligned}
$$

Clearly, the tensors $R, C, G$ and $E \wedge F$ are generalized curvature tensors. For a symmetric $(0,2)$-tensor $E$ we define the $(0,4)$-tensor $\bar{E}$ by $\bar{E}=\frac{1}{2} E \wedge E$. We have $\bar{g}=G=\frac{1}{2} g \wedge g$ and

$$
C=R-\frac{1}{n-2} g \wedge S+\frac{\kappa}{(n-2)(n-1)} G .
$$

We also have (see e.g. [10], eq. (24))

$$
Q(E, E \wedge F)=-Q(F, \bar{E})
$$

Relations (11) and (12) give

$$
Q(g, C)=Q(g, R)+\frac{1}{n-2} Q(S, G)
$$

According to [9], for a symmetric $(0,2)$-tensor $E$ and a $(0, k)$-tensor $T, k \geq 2$, we define their Kulkarni-Nomizu product $E \wedge T$ by

$$
\begin{aligned}
(E \wedge T)\left(X_{1}, X_{2}, X_{3}, X_{4} ; Y_{3}, \ldots, Y_{k}\right) & \\
= & E\left(X_{1}, X_{4}\right) T\left(X_{2}, X_{3}, Y_{3}, \ldots, Y_{k}\right)+E\left(X_{2}, X_{3}\right) T\left(X_{1}, X_{4}, Y_{3}, \ldots, Y_{k}\right) \\
& \quad-E\left(X_{1}, X_{3}\right) T\left(X_{2}, X_{4}, Y_{3}, \ldots, Y_{k}\right)-E\left(X_{2}, X_{4}\right) T\left(X_{1}, X_{3}, Y_{3}, \ldots, Y_{k}\right) .
\end{aligned}
$$

Using the above definitions we can prove the following

Lemma 2.1 ([9], [18]). Let $E_{1}, E_{2}$ and $F$ be symmetric $(0,2)$-tensors at a point $x$ of a semi-Riemannian manifold $(M, g), n \geq 3$. Then at $x$ we have

$$
E_{1} \wedge Q\left(E_{2}, F\right)+E_{2} \wedge Q\left(E_{1}, F\right)=-Q\left(F, E_{1} \wedge E_{2}\right) .
$$

If $E=E_{1}=E_{2}$ then

$$
E \wedge Q(E, F)=-Q(F, \bar{E})
$$

3. Hypersurfaces in conformally flat spaces. Let $M, n \geq 3$, be a connected hypersurface in a semi-Riemannian manifold $\left(N, g^{N}\right)$ and let $g$ be the metric tensor induced on $M$ from $g^{N}$. We denote by $\nabla, \nabla^{N}$, the Levi-Civita connections corresponding to the metric tensors $g$ and $g^{N}$, respectively. Similarly, we denote by $R$ and $R^{N}$ the RiemannChristoffel curvature tensors of $(M, g)$ and $\left(N, g^{N}\right)$, respectively. Let $\xi$ be a local unit normal vector field on $M$ in $N$ and let $\varepsilon=g^{N}(\xi, \xi)= \pm 1$. We can write the Gauss formula and the Weingarten formula of $(M, g)$ in $\left(N, g^{N}\right)$ in the form: $\nabla_{X}^{N} Y=\nabla_{X} Y+\varepsilon H(X, Y) \xi$ 
and $\nabla_{X}^{N} \xi=-\mathcal{A} X$, respectively, where $X, Y$ are vector fields tangent to $M, H$ is the second fundamental tensor of $(M, g)$ in $\left(N, g^{N}\right)$ and $\mathcal{A}$ is the shape operator. Let $x^{r}=x^{r}\left(y^{k}\right)$ be the local parametric expression of $(M, g)$ in $\left(N, g^{N}\right)$, where $y^{k}$ and $x^{r}$ are local coordinates of $M$ and $N$, respectively, and $h, i, j, k \in\{1,2, \ldots, n\}$ and $p, r, t, u \in\{1,2, \ldots, n+1\}$. The Gauss equation of $(M, g)$ in $\left(N, g^{N}\right)$ has the form

$$
R_{h i j k}=R_{p r t u}^{N} B_{h}^{p} B_{i}^{r} B_{j}{ }^{t} B_{k}{ }^{u}+\varepsilon\left(H_{h k} H_{i j}-H_{h j} H_{i k}\right), \quad B_{k}^{r}=\frac{\partial x^{r}}{\partial y^{k}},
$$

where $R_{p r t u}^{N}, R_{h i j k}$ and $H_{h k}$ are the local components of the tensors $R^{N}, R$ and $H$, respectively.

Let now $\left(N, g^{N}\right)$ be a conformally flat space. We have ([11], Section 4)

$$
\begin{aligned}
C_{h i j k} & =\mu G_{h i j k}+\varepsilon \bar{H}_{h i j k}+\frac{\varepsilon}{n-2}\left(g \wedge\left(H^{2}-\operatorname{tr}(H) H\right)\right)_{h i j k}, \\
\mu & =\frac{1}{(n-2)(n-1)}\left(\kappa-2 \widetilde{S}_{r t} B_{h}^{r} B_{k}^{t} g^{h k}+\widetilde{\kappa}\right),
\end{aligned}
$$

where $\widetilde{S}_{r t}$ are the local components of the Ricci tensor $\widetilde{S}$ of the ambient space, $G_{h i j k}$ are the local components of the tensor $G$ and $\widetilde{\kappa}$ and $\kappa$ are the scalar curvatures of $\left(N, g^{N}\right)$ and $(M, g)$, respectively. From (16) we get

$$
\begin{aligned}
C \cdot H= & \frac{\varepsilon}{n-2}\left(Q\left(g, H^{3}\right)+(n-3) Q\left(H, H^{2}\right)\right. \\
& \left.-\operatorname{tr}(H) Q\left(g, H^{2}\right)\right)+\mu Q(g, H), \\
C \cdot H^{2}= & \varepsilon\left(Q\left(H, H^{3}\right)+\frac{1}{n-2}\left(Q\left(g, H^{4}\right)-\operatorname{tr}(H) Q\left(g, H^{3}\right)\right.\right. \\
& \left.\left.-\operatorname{tr}(H) Q\left(H, H^{2}\right)\right)\right)+\mu Q\left(g, H^{2}\right) .
\end{aligned}
$$

THEOREM 3.1. Let $M, \operatorname{dim} M \geq 4$, be a hypersurface in a conformally flat semi-Riemannian manifold.

(i) ([14], Theorem 4.1) $M$ is quasi-umbilical if and only if it is a conformally flat manifold.

(ii) ([16], Theorem 3.1) If $M$ is 2-quasi-umbilical then it is a manifold with pseudosymmetric Weyl tensor.

4. Hypersurfaces in spaces of constant curvature. Let $M$ be a hypersurface in $N_{s}^{n+1}(c), n \geq 4$. Now (15) and (17) read

$$
\begin{aligned}
R_{h i j k} & =\varepsilon \bar{H}_{h i j k}+\frac{\widetilde{\kappa}}{n(n+1)} G_{h i j k}, \\
\mu & =\frac{1}{n-2}\left(\frac{\kappa}{n-1}-\frac{\widetilde{\kappa}}{n+1}\right),
\end{aligned}
$$

respectively. Contracting (20) with $g^{i j}$ and $g^{k h}$ we obtain

$$
\begin{aligned}
S_{h k} & =\varepsilon\left(\operatorname{tr}(H) H_{h k}-H_{h k}^{2}\right)+\frac{(n-1) \widetilde{\kappa}}{n(n+1)} g_{h k}, \\
\kappa & =\varepsilon\left((\operatorname{tr}(H))^{2}-\operatorname{tr}\left(H^{2}\right)\right)+\frac{(n-1) \widetilde{\kappa}}{n+1},
\end{aligned}
$$


respectively, where $\kappa$ is the scalar curvature of $M, \operatorname{tr}(H)=g^{h k} H_{h k}, \operatorname{tr}\left(H^{2}\right)=g^{h k} H_{h k}^{2}$ and $S_{h k}$ are the local components of the Ricci tensor $S$ of $M$. Further, let $A$ be the $(0,2)$-tensor on $M$ defined by

$$
A=H^{3}-\operatorname{tr}(H) H^{2}+\frac{\varepsilon \kappa}{n-1} H .
$$

From Theorem 5.1 of [12] it follows that $A$, defined by (24), vanishes on the subset $\mathcal{U}_{H}$ of any quasi-Einstein Ricci-semisymmetric hypersurface $M$ in $\mathbb{E}_{s}^{n+1}, n \geq 4$. It is also known ([10], Theorem 5.1) that $A=0$ on the subset $\mathcal{U}_{H}$ of a hypersurface $M$ in $N_{s}^{n+1}(c), n \geq 4$, if and only if, on $\mathcal{U}_{H}$, we have

$$
R \cdot C-C \cdot R=\frac{1}{n-2} Q(S, R)+\frac{(n-1) \widetilde{\kappa}}{(n-2) n(n+1)} Q(g, R) .
$$

Examples of hypersurfaces with nonzero tensor $A$ are given in [10]. Further, on any hypersurface $M$ in $N_{s}^{n+1}(c), n \geq 4$, we have ([10], Theorem 3.1):

$$
\begin{aligned}
R \cdot C= & Q(S, R)-\frac{(n-2) \widetilde{\kappa}}{n(n+1)} Q(g, R) \\
& -\frac{(n-3) \widetilde{\kappa}}{(n-2) n(n+1)} Q(S, G)+\frac{1}{n-2} g \wedge Q(H, A), \\
C \cdot R= & \frac{n-3}{n-2} Q(S, R)-\frac{\left(n^{2}-3 n+3\right) \widetilde{\kappa}}{(n-2) n(n+1)} Q(g, R) \\
& -\frac{(n-3) \widetilde{\kappa}}{(n-2) n(n+1)} Q(S, G)+\frac{1}{n-2} H \wedge Q(g, A) .
\end{aligned}
$$

Proposition 4.1. If $M$ is a pseudosymmetric hypersurface in $N_{s}^{n+1}(c), n \geq 4$, then on $\mathcal{U}_{H} \subset M$ we have (4) with

$$
\psi=\frac{1}{2}\left(\operatorname{tr}\left(H^{2}\right)-(\operatorname{tr}(H))^{2}\right)
$$

Proof. Since $M$ is a pseudosymmetric manifold, in view of Theorem 1.2(iii), on $\mathcal{U}_{H}$ we have $\operatorname{rank} H=2$. Now, using Lemma 2.1(i) of [8], we get our assertion.

REMARK 4.1. Examples of hypersurfaces in $N_{s}^{n+1}(c), n \geq 4$, with $\operatorname{rank} H=2$ were found in [11].

THEOREM 4.1. If $M$ is a hypersurface in $N_{s}^{n+1}(c), n \geq 4$, satisfying on $\mathcal{U}_{H} \subset M$

$$
R \cdot C=L Q(g, C)
$$

where $L$ is some function on $\mathcal{U}_{H}$, then rankH $=2$ and (4), i.e. (6) with $\rho=0$, hold on $\mathcal{U}_{H}$.

Proof. On $\mathcal{U}_{H}$, by (28), we have

$$
\begin{aligned}
(R \cdot C)\left(X_{1}, X_{2}, X_{3}, X_{4} ; X_{5}, X_{6}\right)+(R \cdot C) & \left(X_{3}, X_{4}, X_{5}, X_{6} ; X_{1}, X_{2}\right) \\
& +(R \cdot C)\left(X_{5}, X_{6}, X_{1}, X_{2}, X_{3}, X_{4}\right)=0,
\end{aligned}
$$


where $X_{1}, \ldots, X_{6}$ are vector fields tangent to $\mathcal{U}_{H}$. Now on $\mathcal{U}_{H}$ Proposition 5.1 of [18] implies (6) and

$$
R \cdot S=\frac{\widetilde{\kappa}}{n(n+1)} Q(g, S)+\rho Q(g, H) .
$$

On the other hand, from (28), in view of Theorem 1.2(i) and (ii), it follows that (5) holds on $\mathcal{U}_{H}$, which in view of Theorem $1.2\left(\right.$ iii) implies $\operatorname{rank} H=2$ on $\mathcal{U}_{H}$. Further, from (5), by a suitable contraction, we get (8). This, together with (29), gives $\rho=0$. Thus our theorem is proved.

5. Hypersurfaces satisfying $H^{3}=\operatorname{tr}(H) H^{2}+\psi H+\rho g$. Let $M$ be a hypersurface in $N_{s}^{n+1}(c), n \geq 4$, satisfying (6) on $\mathcal{U}_{H} \subset M$. By making use of (6), (24) turns into

$$
A=\left(\frac{\varepsilon \kappa}{n-1}+\psi\right) H+\rho g, \quad \rho=\frac{1}{n}\left(\operatorname{tr}(A)-\left(\frac{\varepsilon \kappa}{n-1}+\psi\right) \operatorname{tr}(H)\right) .
$$

Further, we set on $\mathcal{U}_{H}$

$$
\begin{aligned}
& \beta_{1}=\frac{\varepsilon}{n-2}(\psi+(n-2) \varepsilon \mu), \\
& \beta_{2}=\varepsilon \mu \operatorname{tr}(H)+\frac{1}{n-2}(\psi \operatorname{tr}(H)+(n-3) \rho), \\
& \beta_{3}=\beta_{2}-\varepsilon \beta_{1} \operatorname{tr}(H), \\
& \beta_{4}=\frac{\kappa}{n-1}+\varepsilon \psi-\frac{\left(n^{2}-3 n+3\right) \widetilde{\kappa}}{n(n+1)}, \\
& \beta_{5}=\beta_{1}-\frac{(n-3) \widetilde{\kappa}}{n(n+1)} .
\end{aligned}
$$

Proposition 5.1. If $M$ is a hypersurface in $N_{s}^{n+1}(c), n \geq 4$, satisfying (6) on $\mathcal{U}_{H} \subset M$, for some functions $\psi$ and $\rho$ on $\mathcal{U}_{H}$, then on $\mathcal{U}_{H}$ we have

$$
\begin{aligned}
& R \cdot C=Q(S, R)-\frac{(n-2) \widetilde{\kappa}}{n(n+1)} Q(g, R)+\alpha_{2} Q(S, G)+\frac{\rho}{n-2} Q(H, G), \\
& C \cdot R=\frac{n-3}{n-2} Q(S, R)+\alpha_{1} Q(g, R)+\alpha_{2} Q(S, G), \\
& (n-2)(R \cdot C-C \cdot R)=\left(\frac{(n-1) \widetilde{\kappa}}{n(n+1)}-\frac{\kappa}{n-1}-\varepsilon \psi\right) Q(g, R) \\
& \quad+Q(S, R)+\rho Q(H, G), \\
& C \cdot C=\frac{n-3}{n-2} Q(S, R)+\alpha_{1} Q(g, R) \\
& \quad+\frac{1}{n-2}\left(\left(\alpha_{1}-\alpha_{2}\right) Q(S, G)+\frac{n-3}{n-2} \rho Q(H, G)\right), \\
& \alpha_{1}=\frac{1}{n-2}\left(\frac{\kappa}{n-1}+\varepsilon \psi-\frac{\left(n^{2}-3 n+3\right) \widetilde{\kappa}}{n(n+1)}\right)=\frac{1}{n-2} \beta_{4}, \\
& \alpha_{2}=-\frac{(n-3) \widetilde{\kappa}}{(n-2) n(n+1)} .
\end{aligned}
$$

Proof. By making use of (14) and (30), (25) yields (32). Applying now (6) to (26) and using (14) and (30), we get (33). Subtracting (32) from (33) we easily find (34). The 
relations (6) and (24) yield

$$
\begin{aligned}
& H^{3}-\operatorname{tr}(H) H^{2}=\psi H+\rho g, \\
& H^{4}-\operatorname{tr}(H) H^{3}=\psi H^{2}+\rho H,
\end{aligned}
$$

respectively. Applying (37) to (18) and (19) we obtain

$$
\begin{aligned}
C \cdot H= & \left(\mu+\frac{\varepsilon \psi}{n-2}\right) Q(g, H)+\frac{(n-3) \varepsilon}{n-2} Q\left(H, H^{2}\right), \\
C \cdot H^{2}= & \frac{(n-3) \varepsilon t r(H)}{n-2} Q\left(H, H^{2}\right)+\left(\mu+\frac{\varepsilon}{n-2} \psi\right) Q\left(g, H^{2}\right) \\
& -\frac{(n-3) \varepsilon \rho}{n-2} Q(g, H),
\end{aligned}
$$

respectively. Using (21), (22), (31), (38), and (39) we find

$$
\begin{aligned}
C \cdot S & =\varepsilon C \cdot\left(\operatorname{tr}(H) H-H^{2}\right)+\frac{(n-1) \widetilde{\kappa}}{n(n+1)} C \cdot g \\
& =-\varepsilon \beta_{1} Q\left(g, H^{2}\right)+\beta_{2} Q(g, H) .
\end{aligned}
$$

Applying (22) to (39) and using (31), we get

$$
C \cdot S=\beta_{3} Q(g, H)+\beta_{1} Q(g, S) .
$$

Using (11), (14), (30), (33), (36) and (41) we find

$$
\begin{aligned}
(n-2) C \cdot C= & (n-2) C \cdot R-g \wedge(C \cdot S) \\
= & (n-2) C \cdot R-g \wedge\left(\beta_{3} Q(g, H)+\beta_{1} Q(g, S)\right) \\
= & (n-3) Q(S, R)+(n-2) \alpha_{1} Q(g, R) \\
& +\left(\beta_{1}-\frac{(n-3) \widetilde{\kappa}}{n(n+1)}\right) Q(S, G)+\beta_{3} Q(H, G) .
\end{aligned}
$$

An application of (20) and (22) and Lemma 2.1 leads to the identity

$$
\begin{aligned}
H \wedge Q\left(g, \varepsilon H^{2}\right)-g \wedge Q(H, S) & =H \wedge Q(g, \varepsilon \operatorname{tr}(H) H)-H \wedge Q(g, S)-g \wedge Q(H, S) \\
& =\operatorname{tr}(H) Q(g, R)-(H \wedge Q(g, S)+g \wedge Q(H, S)) \\
& =\operatorname{tr}(H) Q(g, R)+Q(S, g \wedge H) .
\end{aligned}
$$

Applying this and (31) to (42) we get

$$
(n-2) C \cdot C=(n-3) Q(S, R)+\beta_{3} Q(H, G)+\beta_{4} Q(g, R)+\beta_{5} Q(S, G),
$$

which turns into (35), by making use of (21) and (31). Our proposition is thus proved.

Proposition 5.2. If $M$ is a hypersurface in $N_{s}^{n+1}(c), n \geq 4$, satisfying (9) on $\mathcal{U}_{H} \subset M$, for some functions $L_{1}, L_{2}$ and $L_{3}$, then (6) holds on $\mathcal{U}_{H}$. Moreover, at every $x \in \mathcal{U}_{H}$ we have:

(i) $L_{1}=\frac{n-3}{n-2}, L_{2}=\alpha_{1}$ and $L_{3}=\alpha_{2}$, or

(ii) $L_{1} \neq \frac{n-3}{n-2}, \operatorname{rank}\left(S-\alpha_{0} g\right)=1, \rho=0$, and in consequence, (4) and (8), or

(iii) $L_{1} \neq \frac{n-3}{n-2}, \operatorname{rank}\left(S-\alpha_{0} g\right)>1,(5)$, and in consequence, $\operatorname{rank} H=2$, (4) and $\rho=0$, 
where

$$
\alpha_{0}=-\frac{(n-2)\left(L_{2}-\alpha_{1}\right)}{(n-2) L_{1}-(n-3)} .
$$

Proof. In view of Corollary 4.1 of [10], (9) implies (6) on $\mathcal{U}_{H}$. Further, Proposition 5.1 yields (33), which together with (9) leads to

$$
\left(L_{1}-\frac{n-3}{n-2}\right) Q(S, R)+\left(L_{2}-\alpha_{1}\right) Q(g, R)+\left(L_{3}-\alpha_{2}\right) Q(S, G)=0 .
$$

(i) We assume that $L_{1}=\frac{n-3}{n-2}$ at $x$. Now (44) reduces to

$$
\left(L_{2}-\alpha_{1}\right) Q(g, R)+\left(L_{3}-\alpha_{2}\right) Q(S, G)=0 .
$$

In addition, we suppose that $L_{2} \neq \alpha_{1}$ at $x$. Thus (45) yields $Q(g, R)+\alpha_{3} Q(S, G)=0$, for some $\alpha_{3} \in \mathbb{R}$, which by (12), turns into $Q\left(g, R-\alpha_{3} g \wedge S\right)=0$. This gives $R-\alpha_{3} g \wedge S=\alpha_{4} G$, for some $\alpha_{4} \in \mathbb{R}$. But the last relation, in a standard way, implies $C=0$, a contradiction. Therefore we have $L_{2}=\alpha_{1}$. Now (45) reduces to $\left(L_{3}-\alpha_{2}\right) Q(S, G)=0$, whence $L_{3}=\alpha_{2}$.

We assume now that: $L_{1} \neq \frac{n-3}{n-2}$ at $x$. Thus (44) turns into

$$
Q\left(S-\alpha_{0} g, R\right)+\alpha_{5} Q(S, G)=0,
$$

where $\alpha_{0}$ is defined by (43) and $\alpha_{5} \in \mathbb{R}$. Since $Q(S, G)=Q\left(S-\alpha_{0} g, G\right)=0$, (46) yields

$$
Q\left(S-\alpha_{0} g, R+\alpha_{5} G\right)=0 .
$$

(ii) Let $\operatorname{rank}\left(S-\alpha_{0} g\right)=1$ at $x$. Applying this to (22) we obtain

$$
H^{2}=\operatorname{tr}(H) H+\alpha_{6} g+\alpha_{7} w \otimes w, \quad w \in T_{x}^{*} M,
$$

for some $\alpha_{6}, \alpha_{7} \in \mathbb{R}$, where $\alpha_{7} \neq 0$. From (48) it follows that

$$
H_{i j}^{3}=\operatorname{tr}(H) H_{i j}^{2}+\alpha_{6} H_{i j}+\alpha_{7} w^{r} H_{r i} w_{j},
$$

where $w^{r}=g^{r j} w_{j}$ and $w_{j}$ are the local components of $w$. But (49) implies $w^{r} H_{r j}=\alpha_{8} w_{j}$, for some $\alpha_{8} \in \mathbb{R}$. Applying the last equation and (48) to (49), we get

$$
H^{3}=\left(\operatorname{tr}(H)+\alpha_{8}\right) H^{2}+\left(\alpha_{6}-\alpha_{8} \operatorname{tr}(H)\right) H-\alpha_{6} \alpha_{8} g .
$$

Comparing this with (6) we obtain

$$
\alpha_{8} H^{2}+\left(\alpha_{6}-\alpha_{8} \operatorname{tr}(H)-\psi\right) H-\left(\alpha_{6} \alpha_{8}+\rho\right) g=0 .
$$

Since $x \in \mathcal{U}_{H}$, we have $\alpha_{8}=0, \alpha_{6}=\psi$ and $\rho=0$. Thus (6) reduces to (4). But (4), in view of Theorem 1.2(iv), implies (8).

(iii) Let $\operatorname{rank}\left(S-\alpha_{0} g\right)>1$ at $x$. Now (47), in view of Lemma 4.2 of [10], implies

$$
R+\alpha_{5} G=\frac{\alpha_{9}}{2}\left(S-\alpha_{0} g\right) \wedge\left(S-\alpha_{0} g\right)
$$

for some $\alpha_{9} \in \mathbb{R}-\{0\}$. The last relation implies (7). Now Theorem 1.2(ii) and (iii) completes the proof.

A consequence of the last proposition is the following

THEOREM 5.1. Let $M$ be a hypersurface in $N_{s}^{n+1}(c), n \geq 4$, and let $V$ be the set of all points of $\mathcal{U}_{H} \subset M$ at which the tensor $R \cdot S-\frac{\widetilde{\kappa}}{n(n+1)} Q(g, S)$ is nonzero. Then on $V$ the condition (6) is equivalent to (33) and (36). 
We finish this section with results on hypersurfaces with pseudosymmetric Weyl tensor.

THEOREM 5.2. If $M$ is a hypersurface in $N_{s}^{n+1}(c), n \geq 4$, satisfying (3) and (6) on $\mathcal{U}_{H} \subset M$ then on this set we have (4), i.e. (6) with $\rho=0$, (5), (27) and

$$
L_{C}=\frac{n-3}{2(n-2)}\left(\frac{\widetilde{\kappa}}{n+1}-\frac{\kappa}{n-1}\right) \text {. }
$$

Proof. (35), by (3) and (11), turns into

$$
\begin{aligned}
Q(S, R)= & \frac{1}{n-3}\left((n-2) L_{C}-\frac{\kappa}{n-1}-\varepsilon \psi+\frac{\left(n^{2}-3 n+3\right) \widetilde{\kappa}}{n(n+1)}\right) Q(g, R) \\
& +\frac{1}{(n-3)(n-2)}\left((n-2) L_{C}-\frac{\kappa}{n-1}-\varepsilon \psi+\frac{\left(n^{2}-4 n+6\right) \widetilde{\kappa}}{n(n+1)}\right) Q(S, G) \\
& -\frac{\rho}{n-2} Q(H, G) .
\end{aligned}
$$

Now (32) and (51) yield

$$
R \cdot C=\frac{1}{n-3}\left((n-2) L_{C}-\frac{\kappa}{n-1}-\varepsilon \psi+\frac{(2 n-3) \widetilde{\kappa}}{n(n+1)}\right)\left(Q(g, R)+\frac{1}{n-2} Q(S, G)\right),
$$

which, by (13), turns into

$$
R \cdot C=\frac{1}{n-3}\left((n-2) L_{C}-\frac{\kappa}{n-1}-\varepsilon \psi+\frac{(2 n-3) \widetilde{\kappa}}{n(n+1)}\right) Q(g, C) .
$$

This, in view on Theorem 4.1(i) and (ii), implies

$$
\begin{aligned}
R \cdot R & =\frac{1}{n-3}\left((n-2) L_{C}-\frac{\kappa}{n-1}-\varepsilon \psi+\frac{(2 n-3) \widetilde{\kappa}}{n(n+1)}\right) Q(g, R), \\
\frac{\widetilde{\kappa}}{n(n+1)} & =\frac{1}{n-3}\left((n-2) L_{C}-\frac{\kappa}{n-1}-\varepsilon \psi+\frac{(2 n-3) \widetilde{\kappa}}{n(n+1)}\right),
\end{aligned}
$$

which yields (5) and

$$
L_{C}=\frac{1}{n-2}\left(\frac{\kappa}{n-1}-\frac{\widetilde{\kappa}}{n+1}+\varepsilon \psi\right) .
$$

Since (5) holds on $\mathcal{U}_{H}$, Proposition 4.1 implies (4) and (27) on $\mathcal{U}_{H}$. Further, applying (23) and (27) into (51) we obtain (50), which completes the proof.

TheOREM 5.3. Every 2-quasi-umbilical hypersurface $M$ in $N_{s}^{n+1}(c), n \geq 4$, satisfying (6) on $\mathcal{U}_{H} \subset M$, is 2-cylindrical on this set.

Proof. Since $M$ is a 2-quasi-umbilical hypersurface, in view of Theorem 3.1(ii), (3) holds on $\mathcal{U}_{H}$. Using now Theorem 4.1 (iv) and Theorem 5.2 on $\mathcal{U}_{H}$ we get rank $H=2$. Further, from (2) we have $\operatorname{rank} B=2$, where $B=H-\alpha g$. The last two equations, in view of Lemma 2.1 of [8] (see eq. (19) of [8]), yield $\operatorname{tr}(H) \bar{H}=H \wedge H^{2}$ and $\operatorname{tr}(B) \bar{B}=B \wedge B^{2}$, respectively. From these relations, by suitable contraction, we find $\alpha\left(H^{2}+\alpha_{1} H+\alpha_{2} g\right)=0$, where $\alpha_{1}$ and $\alpha_{2}$ are some functions on $\mathcal{U}_{H}$. But from the last equation it follows that $\alpha=0$ on $U_{H}$, which completes the proof. 


\section{References}

[1] K. Arslan, R. Deszcz, R. Ezentaș, C. Murathan, and C. Ozgür, On pseudosymmetry type hypersurfaces of semi-Euclidean spaces, Demonstr. Math. 36 (2003), 971-984.

[2] K. Arslan, R. Deszcz, and Ș. Yaprak, On Weyl pseudosymmetric hypersurfaces, Colloq. Math. 72 (1997), 353-361.

[3] M. Belkhelfa, R. Deszcz, M. Głogowska, M. Hotloś, D. Kowalczyk, and L. Verstraelen, On some type of curvature conditions, in: Banach Center Publ. 57, Inst. Math., Polish Acad. Sci., 2002, 179-194.

[4] F. Defever, R. Deszcz, P. Dhooghe, L. Verstraelen, and Ș. Yaprak, On Riccipseudosymmetric hypersurfaces in spaces of constant curvature, Results Math. 27 (1995), $227-236$.

[5] R. Deszcz, On pseudosymmetric spaces, Bull. Soc. Belg. Math., Ser. A, 44 (1992), 1-34.

[6] R. Deszcz, On certain classes of hypersurfaces in spaces of constant curvature, in: Geometry and Topology of Submanifolds, VIII, World Sci., River Edge, NJ, 1996, 101-110.

[7] R. Deszcz, Pseudosymmetric hypersurfaces in spaces of constant curvature, Tensor (N.S.) 58 (1997), 253-269.

[8] R. Deszcz, On some Akivis-Goldberg type manifolds, Publ. Inst. Math. (Beograd) (N.S.) 74(88) (2003), 71-83.

[9] R. Deszcz and M. Głogowska, Some nonsemisymmetric Ricci-semisymmetric warped product hypersurfaces, Publ. Inst. Math. (Beograd) (N.S.) 72(86) (2002), 81-93.

[10] R. Deszcz, M. Głogowska, M. Hotloś, and L. Verstraelen, On some generalized Einstein metric conditions on hypersurfaces in semi-Riemannian space forms, Colloq. Math. 96 (2003), 149-166.

[11] R. Deszcz and M. Hotloś, On hypersurfaces with type number two in spaces of constant curvature, Ann. Univ. Sci. Budap. Rolando Eötvös, Sect. Math. 46 (2003), 19-34.

[12] R. Deszcz, M. Hotloś, and Z. Șentürk, On curvature properties of quasi-Einstein hypersurfaces in semi-Euclidean spaces, Soochow J. Math. 27 (2001), 375-389.

[13] R. Deszcz and K. Sawicz, On a class of hypersurfaces in semi-Euclidean spaces, Dept. Math. Agricultural Univ. Wrocław, Ser. A, Theory and Methods, Report No. 107, 2002.

[14] R. Deszcz and L. Verstraelen, Hypersurfaces of semi-Riemannian conformally flat manifolds, in: Geometry and Topology of Submanifolds, III, World Sci., River Edge, NJ, 1991, 131-147.

[15] R. Deszcz, L. Verstraelen, and Ș. Yaprak, On hypersurfaces with pseudosymmetric Weyl tensor, in: Geometry and Topology of Submanifolds, VIII, World Sci., River Edge, NJ, 1996, 111-120.

[16] R. Deszcz, L. Verstraelen, and Ș. Yaprak, On 2-quasi-umbilical hypersurfaces in conformally flat spaces, Acta Math. Hungarica 78 (1998), 45-57.

[17] R. Deszcz, L. Verstraelen, and Ș. Yaprak, Hypersurfaces with pseudosymmetric Weyl tensor in conformally flat manifolds, in: Geometry and Topology of Submanifolds, IX, World Sci., River Edge, NJ, 1999, 108-117.

[18] M. Głogowska, On a curvature characterization of Ricci-pseudosymmetric hypersurfaces, Acta Math. Scientia 24B (2004), 361-375.

[19] K. Sawicz, Examples of hypersurfaces in Euclidean spaces with three distinct principal curvatures, to appear. 